\title{
Clostridioides difficile in Outpatients: Application of a Diagnostic Algorithm Recommended by the European Society of Clinical Microbiology and Infectious Diseases
}

\author{
Ralf Ignatius $^{1,2^{*}}$, Robert Neuber ${ }^{1}$, Heike Kietzmann ${ }^{1}$, Christiane Berg ${ }^{1}$, Thilo Wenzel ${ }^{1}$, Jörg Fuhrmann $^{1}$ \\ and Michael Müller ${ }^{1}$ \\ ${ }^{1}$ MVZ Labor 28, Mecklenburgische Str. 28, 14197 Berlin, Germany \\ ${ }^{2}$ Department of Microbiology and Infection Immunology, Charité - Universitätsmedizin Berlin, Campus Benjamin Franklin, \\ Hindenburgdamm 30, 12200 Berlin, Germany
}

Received: 03 Aug 2019; accepted: 09 Aug 2019

\begin{abstract}
This study aimed at evaluating in outpatients an algorithm for the laboratory diagnosis of Clostridioides (Clostridium) difficile infection (CDI), i.e., enzyme immunoassays (EIAs) detecting bacterial glutamate dehydrogenase $(\mathrm{GDH})$ and toxin $\mathrm{A} / \mathrm{B}$, followed by polymerase chain reaction (PCR) analyses of samples with discordant EIA results.

In total, 9802 examinations of stool samples by GDH and toxin EIAs performed in 7263 outpatients and 488 inpatients were analyzed retrospectively. Samples with discordant EIA results had been tested by a commercially available PCR assay detecting genes of the $C$. difficile-specific triose phosphate isomerase (tpi) and toxin B (tcdB). Concordant EIA results (686 C. difficile-positive, 8121 negative) were observed for 8807 (89.8\%; 95\% CI, 89.2$90.4 \%$ ) samples. Of 958 samples with discordant EIA results, 895 were analyzed using PCR and 580 of 854 GDHpositive/borderline, toxin-negative samples $(67.9 \%$; 95\% CI, 64.7-71.0\%) were positive for tpi and $t c d B$, while 274 samples $(32.1 \%$; 95\% CI, 29.0-35.3\%) were $t c d B$-negative. In contrast, 35 of 41 GDH-negative, toxin-positive/borderline samples $(85.4 \%$; 95\% CI, 71.2-93.5\%) were $t c d B$-negative. Still, 6 samples $(14.6 \%$; 95\% CI, 6.5-28.8\%) yielded positive PCR results for both genes.

In conclusion, around $90 \%$ of the samples were analyzed appropriately by only applying EIAs. Approximately one third of the PCR-analyzed samples were $t c d B$-negative; thus, patients most likely did not require CDI treatment.

Keywords: Clostridioides difficile, Clostridium difficile, EIA, PCR, algorithm, ESCMID
\end{abstract}

\section{Introduction}

Clostridioides (Clostridium) difficile infection (CDI) comprises a wide range of gastrointestinal symptoms, and consumption of antibiotics usually precedes CDI [1]. While toxigenic culture (TC) and cell cytotoxicity neutralization assay are the gold standard tests for the laboratory diagnosis of CDI $[2,3]$, at present, the routine mainly consists of the detection of the bacterial glutamate dehydrogenase (GDH) and toxins $\mathrm{A} / \mathrm{B}$ by enzyme immunoassays (EIAs) [2, 3]. A major problem of this strategy, however, is the limited sensitivity of most commercial toxin EIAs. This obstacle may be overcome by the additional use of highly sensitive and specific nucleic acid amplification tests (NAAT), and various algorithms for the laboratory diagnosis of CDI involving these assays have been evaluated, but mainly with samples of hospitalized patients $[2,3]$.

The European Society of Clinical Microbiology and Infectious Diseases (ESCMID) has recently recommended 2 algorithmic strategies [2]: algorithm A starting with NAAT or GDH EIA, followed by toxin EIA, and optionally TC or NAAT (in case the GDH EIA was used initially) to analyze NAAT/GDH-positive, toxin-negative samples; and algorithm

*Author for correspondence: MVZ Labor 28, Mecklenburgische Str. 28, 14197 Berlin; Tel.: 030-82093262; E-mail: ignatius@labor28.de
B where GDH and toxin EIAs are performed simultaneously, followed by NAAT or TC as a second optional step. This algorithm has previously been evaluated in a tertiary pediatric population [4] and at a university hospital [5].

The present study aimed at investigating the feasibility of using ESCMID algorithm B under routine conditions for samples of outpatients. In particular, we were interested in the performance of the assays, i.e., the proportion of samples yielding discordant EIA results, since CDI in outpatients may be less severe due to less toxin production, which might lead to increased numbers of toxin-negative samples. Furthermore, we asked how many samples could be considered "most likely uncritical" for lacking $t c d B$-expression.

\section{Materials and Methods}

Patients' Data. All data on GDH/toxin EIAs between February 2017 and March 2019 were extracted from the laboratory information system. In total, 9802 data sets belonging to 7751 patients (7263 outpatients, 93.7\%; 95\% CI, $93.1-94.2 \%$; 488 inpatients mainly from geriatric hospitals, $6.3 \%$; 95\% CI, 5.8-6.9\%) were included.

Enzyme Immunoassays. EIAs (both RIDASCREEN ${ }^{\circledR}$; R-Biopharm AG, Darmstadt, Germany) for the detection of C. difficile $\mathrm{GDH}$ and toxins $\mathrm{A} / \mathrm{B}$ in stool samples were used according to the manufacturer's instructions.

This is an open-access article distributed under the terms of the Creative Commons Attribution-NonCommercial 4.0 International License (https://creativecommons.org/licenses/by-nc/4.0/), which permits unrestricted use, distribution, and reproduction in any medium for non-commercial purposes, provided the original author and source are credited, a link to the CC License is provided, and changes - if any - are indicated. 
Table 1. GDH and toxin EIA data for all stool samples $(n=9802)$, as well as for samples from outpatients only $(n=9151)$

\begin{tabular}{|c|c|c|c|c|c|}
\hline \multirow[t]{2}{*}{ GDH EIA } & \multirow[t]{2}{*}{ Toxin EIA } & \multicolumn{2}{|c|}{ All samples } & \multicolumn{2}{|c|}{ Samples from outpatients } \\
\hline & & $n$ & $\%(95 \%$ CIs $)$ & $n$ & $\%(95 \%$ CIs $)$ \\
\hline \multirow[t]{4}{*}{ Positive } & & 1614 & $16.5(15.7-17.2)$ & 1490 & $16.3(15.5-17.1)$ \\
\hline & Positive & 686 & $7.0(6.5-7.5)$ & 630 & $6.9(6.4-7.4)$ \\
\hline & Negative & 893 & $9.1(8.6-9.7)$ & 829 & $9.1(8.5-9.7)$ \\
\hline & Borderline & 35 & $0.4(0.3-0.5)$ & 31 & $0.4(0.2-0.5)$ \\
\hline \multirow[t]{3}{*}{ Borderline } & & 22 & $0.2(0.2-0.3)$ & 19 & $0.2(0.2-0.3)$ \\
\hline & Positive & 2 & $0.03(0-0.08)$ & 2 & $0.03(0-0.09)$ \\
\hline & Negative & 20 & $0.2(0.1-0.3)$ & 17 & $0.2(0.1-0.3)$ \\
\hline \multirow[t]{4}{*}{ Negative } & & 8166 & $83.4(82.6-84.1)$ & 7642 & $83.5(82.7-84.3)$ \\
\hline & Positive & 35 & $0.4(0.3-0.5)$ & 34 & $0.4(0.3-0.5)$ \\
\hline & Negative & 8121 & $82.8(82.1-83.6)$ & 7598 & $83.0(82.3-83.8)$ \\
\hline & Borderline & 10 & $0.1(0.05-0.2)$ & 10 & $0.1(0.06-0.2)$ \\
\hline
\end{tabular}

Polymerase Chain Reaction (PCR) Assay. Samples with discordant EIA results were tested by a commercially available PCR assay (FluoroType ${ }^{\circledR}$ CDiff, Hain Lifescience, Nehren, Germany), detecting genes of the C. difficile-specific triose phosphate isomerase $(t p i)$ and toxin $\mathrm{B}(t c d B)$, which reliably provides results within around $3 \mathrm{~h}$. The assay was performed according to the manufacturer's instructions.

Statistical Analyses. Data were analyzed by calculation of $95 \%$ confidence intervals $(95 \% \mathrm{CI})$, and statistical significance was determined for "age" by Mann-Whitney test and for "sex" by Fisher's exact test. $P$ values $<0.05$ were considered significant.

Ethics. Since the study was a retrospective analysis of laboratory data collected within around 2 years and no additional investigations were performed other than requested, no ethical approval was applied for. The study is in agreement with the General Data Protection Regulation of the European Union.

\section{Results}

In the study period, 9802 samples were analyzed by GDH and toxin EIAs: 9151 samples $(93.4 \%$; 95\% CI, 92.9-93.8\%) from outpatients and 651 samples $(6.7 \%$; $95 \%$ CI, 6.2-7.2\%) from inpatients. Patients' median age was 62 years (range, $<1-$ 103 years); 5944 samples (60.6\%; 95\% CI, 59.7-61.6\%) were from female patients and 3858 from male patients $(39.4 \%$; 95\% CI, 38.4-40.3\%).

Concordant EIA results, i.e., positivity or negativity in both immunoassays, were obtained for $8807(89.8 \%$; 95\% CI, 89.2-90.4\%) samples, with 686 samples being $C$. difficile-positive and 8121 samples negative (Table 1). Patients tested $C$. difficile-positive by both EIAs were older than patients with negative EIA results (positive: median $=79$ years, range $=4$ 101 years; negative: median $=58$ years, range $<1-102$ years; $P<0.0001)$, while there was no difference regarding sex $(P=0.133)$. Additional 35 GDH-positive, toxin-borderline samples, as well as 2 GDH-borderline, toxin-positive samples also were considered $C$. difficile-positive and not tested using PCR. The remaining 958 samples $(9.8 \%$; 95\% CI, 9.2-10.4\%) yielded discordant EIA results (Table 1), i.e., 913 GDH-positive/borderline samples were toxin-negative, and 45 samples were toxin-positive/borderline but GDH-negative. Almost identical EIA results were obtained when samples from outpatients only were analyzed (Table 1).

Of the 958 samples with discordant EIA results, 895 were further analyzed using PCR, while for 63 samples, either there was not enough material, or the physicians having requested the laboratory diagnostics had declined PCR analysis. Furthermore, samples submitted evidently as controls following therapy of prior CDI, which is not recommended [2], were neither examined using PCR. As expected because of the relatively low sensitivity of toxin EIAs, 580 of 854 GDH-positive/borderline, toxin-negative samples $(67.9 \%$; 95\% CI, 64.7-71.0\%) were positive for both tpi and $t c d B$, while 274 samples (32.1\%; 95\% CI, 29.0-35.3\%) were $t c d B$-negative (Table 2). In contrast, 34 of $41 \mathrm{GDH}$-negative, toxin-positive/borderline samples $(82.9 \%$; 95\% CI, 68.4-91.8\%) analyzed using PCR were negative for both tpi and $t c d B$ and therefore false-positive by the toxin EIA (Table 3). Still, 6 samples (14.6\%; 95\% CI, 6.5-28.8\%) yielded positive PCR results for both genes and had to be considered $C$. difficile-positive. Taken together, however, 309 of 895 samples (34.6\%; 95\% CI, 31.5-37.7\%) lacking $t c d B$ were identified using PCR.

\section{Discussion}

By applying the ESCMID algorithm B, unequivocal EIA results were received for around $90 \%$ of samples. Both GDH and toxin EIAs were positive in $6.9 \%$ of samples from outpatients, which further emphasizes that CDI is a relevant community disease, since patients may develop CDI after discharge from the hospital [6]. Notably, this percentage was higher than that previously observed at a university hospital

Table 3. PCR data of 41 GDH-negative, toxin-positive/borderline stool samples

\begin{tabular}{lccc}
\hline EIA results & \multicolumn{3}{c}{ PCR results } \\
\cline { 2 - 4 } & $t p i+/ t c d B+$ & $t p i+/ t c d B-$ & $t p i-/ t c d B-$ \\
\hline Total & $6^{a}$ & 1 & 34 \\
Toxin-positive & $14.6(6.5-28.8)$ & $2.4(0-13.7)$ & $82.9(68.4-91.8)$ \\
$(n=34)$ & 6 & 1 & 27 \\
$\begin{array}{l}\text { Toxin-borderline } \\
(n=7)\end{array}$ & 0 & 0 & 7 \\
$\quad a_{0}(95 \% \mathrm{CIs})$. & & & \\
\hline
\end{tabular}

Table 2. PCR data of 854 GDH-positive/borderline, toxin-negative stool samples

\begin{tabular}{lccc}
\hline EIA results & PCR results & & \\
\cline { 2 - 4 } & $t p i+/ t c d B+$ & $t p i+/ t c d B-$ & 141 \\
\hline Total & 580 & $16.5(14.2-19.2)$ & 133 \\
GDH-positive $(n=840)$ & $67.9(64.7-71.0)^{a}$ & 139 & $15.6(13.3-18.2)$ \\
GDH-borderline $(n=14)$ & 578 & 2 & 123 \\
$a_{0}(95 \%$ CIs). & 2 & & 10 \\
\hline
\end{tabular}


(4.4\% of 4321 samples; [5]). This difference, however, also might be due to the use of an immunochromatography assay (ICA) in that study as compared to EIAs by us, as the sensitivity of toxin ICAs is slightly lower than that of toxin EIAs [2].

Furthermore, CDI could be excluded with high negative predictive value (NPV) in around one third of the samples by the lack of $t c d B$-expression. Still, 7 GDH-negative but toxinpositive samples had to be considered GDH false-negative, since tpi could be detected by the PCR assay, and 6 of these were additionally $t c d B$-positive. Although we cannot speculate as to whether these samples were derived from true CDI patients, these samples would have been missed in other algorithmic approaches where GDH-negative samples are not further analyzed. Notably, while PCR testing alone might be faster and more sensitive than algorithmic approaches [7], this strategy also might lead to overdiagnosis of CDI, since $t c d B$ expression not always correlates with toxin production [8]. Yet, PCR-positivity/toxin-EIA-negativity of specimens does not exclude CDI [9].

One limitation of our study is the lack of clinical data and of an additional assay to compare PCR results, e.g., TC. The scope of the present study, however, was the evaluation of laboratory workload resulting from PCR analyses in addition to EIAs in outpatients' samples, as well as to determine the proportion of $t c d B$-negative samples. Notably, sensitivity, specificity, and positive and negative predictive values of all assays used for CDI diagnosis depend on the CDI prevalence in the patient population examined [2].

In conclusion, by applying the ESCMID algorithm B, concordant EIA results can be reported in a timely manner for around $90 \%$ of outpatients. Limited additional laboratory work is required to clarify samples with discordant EIA results, and the lack of $t c d B$ expression in around one third of samples may contribute to a rational use of antibiotics in the corresponding patients.

\section{Funding Sources}

No additional funding was received to perform this study.

\section{Authors' Contributions}

RI, HK, CB, TW, JF and MM designed the study; RN and RI analysed the data; all authors wrote the manuscript.

\section{Conflict of Interest}

The authors declare no conflict of interest.

Acknowledgement. We thank the entire staff of our microbiology and molecular biology laboratories for expert technical assistance.

\section{References}

1. Leffler DA, Lamont JT. Clostridium difficile Infection. N Engl J Med. 2015;373(3):287-8.

2. Crobach MJ, Planche T, Eckert C, Barbut F, Terveer EM, Dekkers OM, et al. European Society of Clinical Microbiology and Infectious Diseases: update of the diagnostic guidance document for Clostridium difficile infection. Clin Microbiol Infect. 2016;22(Suppl 4):S63-81.

3. Martinez-Melendez A, Camacho-Ortiz A, Morfin-Otero R, MaldonadoGarza HJ, Villarreal-Trevino L, Garza-Gonzalez E. Current knowledge on the laboratory diagnosis of Clostridium difficile infection. World J Gastroenterol. 2017;23:1552-67.

4. Ota KV, McGowan KL. Clostridium difficile testing algorithms using glutamate dehydrogenase antigen and $C$. difficile toxin enzyme immunoassays with $C$. difficile nucleic acid amplification testing increase diagnostic yield in a tertiary pediatric population. J Clin Microbiol. 2012;50:1185-8.

5. Culbreath K, Ager E, Nemeyer RJ, Kerr A, Gilligan PH. Evolution of testing algorithms at a university hospital for detection of Clostridium difficile infections. J Clin Microbiol. 2012;50:3073-6.

6. Olesen B, Hallberg H, Bangsborg J, Jensen JN, Jarlov JO. A new approach to recognition of Clostridium difficile infections with community onset. Clin Microbiol Infect. 2015;21:e55-6.

7. Novak-Weekley SM, Marlowe EM, Miller JM, Cumpio J, Nomura JH, Vance $\mathrm{PH}$, et al. Clostridium difficile testing in the clinical laboratory by use of multiple testing algorithms. J Clin Microbiol. 2010 Mar;48:889-93.

8. Polage CR, Gyorke CE, Kennedy MA, Leslie JL, Chin DL, Wang S, et al. Overdiagnosis of Clostridium difficile Infection in the Molecular Test Era. JAMA Intern Med. 2015;175:1792-801.

9. Origuen J, Corbella L, Orellana MA, Fernandez-Ruiz M, LopezMedrano F, San Juan R, et al. Comparison of the clinical course of Clostridium difficile infection in glutamate dehydrogenase-positive toxinnegative patients diagnosed by PCR to those with a positive toxin test. Clin Microbiol Infect. 2018;24:414-21. 